\title{
Long-term survival after surgery for pulmonary metastases from colorectal cancer: an observational cohort study
}

\author{
Mamdoh Al-Ameri ${ }^{1,2}$, Michael Persson ${ }^{1,2}$, Per Bergman $^{1,2}$, Anders Franco-Cereceda $^{1,2}$, Ulrik Sartipy ${ }^{1,2}$ \\ ${ }^{1}$ Section of Cardiothoracic Surgery, Karolinska University Hospital, Stockholm, Sweden; ${ }^{2}$ Department of Molecular Medicine and Surgery, \\ Karolinska Institutet, Stockholm, Sweden \\ Contributions: (I) Conception and design: M Al-Ameri, A Franco-Cereceda, U Sartipy; (II) Administrative support: A Franco-Cereceda; (III) Provision \\ of study materials or patients: None; (IV) Collection and assembly of data: M Al-Ameri; (V) Data analysis and interpretation: M Al-Ameri, U Sartipy; \\ (VI) Manuscript writing: All authors; (VII) Final approval of manuscript: All authors. \\ Correspondence to: Ulrik Sartipy, MD, PhD. Section of Cardiothoracic Surgery, Karolinska University Hospital, SE-171 76 Stockholm, Sweden. \\ Email: Ulrik.Sartipy@karolinska.se.
}

\begin{abstract}
Background: Evidence for pulmonary metastasectomy following colorectal cancer (CRC) is scarce. The aim of the study was to investigate long-term survival and identify prognostic factors to aid patient selection.

Methods: We included all patients who underwent pulmonary resections for CRC metastases between January 01, 2004 and December 31, 2015 in a population-based cohort study. The primary outcome measure was all-cause mortality and was ascertained from Swedish national registers. The Kaplan-Meier estimator was used to calculate cumulative survival. We used Cox regression for estimation of hazard ratios (HR) and $95 \%$ confidence intervals (CI) for the association between patient characteristics and survival.

Results: We included 184 patients. The number of procedures per year increased from 1 in 2004 to 34 in 2015. During a median follow-up time of 3.2 years, 36\% (66/184) patients died. Overall survival at 5 years was $60 \%$ (95\% CI: 50-68\%) and was significantly lower compared to an age- and gender-matched Swedish population. Carcinoembryonic antigen (CEA) level was identified as a prognostic factor for mortality in the age and sex-adjusted analysis (HR, 2.46; 95\% CI: 1.15-5.26, $\mathrm{P}=0.020)$.

Conclusions: We found a steady increase in the number of pulmonary metastasectomies after CRC during the study period. We identified prethoracotomy CEA level as a prognostic factor for long-term survival, which was consistent with prior reports. The 5 -year overall survival rate in our study was $60 \%$, which was high in comparison with prior reports. Although our results indicated that current patient selection criteria were reasonable, definitive evidence of efficacy is pending.
\end{abstract}

Keywords: Pulmonary metastasectomy; colorectal cancer (CRC); long-term survival; prethoracotomy carcinoembryonic antigen level (prethoracotomy CEA level)

Submitted Apr 18, 2017. Accepted for publication Sep 08, 2017.

doi: $10.21037 /$ jtd.2017.10.51

View this article at: http://dx.doi.org/10.21037/jtd.2017.10.51

\section{Introduction}

Colorectal cancer (CRC) is the third most commonly diagnosed cancer in males and the second in females, with an estimated global 1.4 million cases and 693,900 deaths annually $(1,2)$. In Sweden approximately 6,000 new cases of CRC are diagnosed every year (3).

About $50 \%$ will have metastatic disease either at diagnosis or during follow-up (4). The two most common sites for metastatic growth are the liver and the lungs affecting roughly $35 \%$ and $5 \%$ to $15 \%$ of patients, respectively $(5,6)$. In recent years, advances have been made in the field of diagnosis, staging and oncologic systemic therapy. The effect can be seen with improved survival in patients with CRC and in patients with liver metastases, whom previously had a reported 5 -year survival of about 
$25 \%$ compared to $50 \%$ today (4). The progress in the management of CRC has made it feasible to perform surgery as a curative treatment in metastatic disease in the liver. In an attempt to achieve improved long-term survival even in patients with metastatic disease in the lung, the practice of pulmonary metastasectomy has become common and widespread. However, the evidence for this practice consists mostly of retrospective trials with variable results (7). Prognostic factors suggested from previous data include disease-free interval (DFI), number and laterality of metastases, hilar and mediastinal lymph nodes ( $t-\mathrm{LN}$ ), previous metastatic disease in the liver, staging of primary tumor, prethoracotomy carcinoembryonic antigen (CEA), K-RAS gene status, carbohydrate antigen 19-9 (CA19-9) and location of the primary tumor (8-11). However, no consensus has been reached as to what factors should be taken into consideration when recommending surgery or not.

This study aims to characterize the practice of lung metastasectomy at Karolinska University Hospital and to identify prognostic variables that might contribute to future pooled data analysis and clinical decision-making.

\section{Methods}

The study was approved by the regional Human Research Ethics Committee, Stockholm, Sweden (Dnr: 2014/129_ $31 / 1)$. The need for informed consent was waived by the Ethics Committee.

\section{Study design}

Observational population-based cohort study.

\section{Patients and outcomes measures}

Patients who underwent surgical resection of pulmonary metastases from CRC at Karolinska University Hospital, Stockholm, Sweden, between January 01, 2004 and December 31, 2015 were included in the study. Karolinska University Hospital is the only referral center for thoracic surgery in Stockholm County and serves approximately two million inhabitants or $20 \%$ of the total Swedish population. Patient data were collected from institutional databases and patient charts. The primary outcome measure was all-cause mortality. Vital status was determined on January 15, 2017 by using the Swedish personal identity number (12) and the continuously updated Swedish population register (13).
The general criteria to accept patients for surgery at multidisciplinary team conferences were in line with clinical practice guidelines $(3,14)$. Patients were in general recommended pulmonary metastasectomy according to the following conditions: radically treated primary tumor, without extra-pulmonary metastasis except synchronous liver metastases amenable to radical treatment, having one or more resectable metastases, and a general condition allowing a safe operation. Radical treatment of the primary tumor and the liver metastases was usually performed before the pulmonary resection.

\section{Definitions}

Comorbidity was defined as any current or prior major medical condition that required ongoing treatment or could influence prognosis e.g., hypertension, atrial fibrillation, coronary artery disease, diabetes, or stroke. The extent of lung resection was divided into two groups: sublobar resection vs. lobectomy. Smoking status was divided into four categories; current, former, never, and unknown. Current smoker was defined as an active smoker or a person who had stopped smoking within one month of surgery. Former smoker was defined as a previous smoker who had stopped smoking more than one month before surgery. Never smoker was defined as a person who had never been an active smoker

\section{Statistical analyses}

Baseline characteristics were described with frequencies and percentages for categorical variables and mean and standard deviation for continuous variables. Person-time in days was counted from the date of pulmonary resection until the date of death or the end of follow-up (January 15, 2017). The Kaplan-Meier method was used to calculate cumulative survival. We used Cox proportional hazards regression with and without multivariable adjustment to estimate hazard ratios (HR) and $95 \%$ confidence intervals (CI) for the association between patient characteristics and all-cause mortality. Statistical analyses were performed using Stata version 14.2 (StataCorp LP, College Station, Texas, USA).

\section{Results}

\section{Patient characteristics}

We included 184 patients with a mean age of 64.8 years and $46 \%$ were women. Patient characteristics are shown in 
Table 1 Patient characteristics in 184 patients who underwent pulmonary metastasectomy after colorectal cancer

\begin{tabular}{|c|c|}
\hline Variable & $\mathrm{N}(\%)$ \\
\hline Age, year, mean (SD) & $64.8(10.2)$ \\
\hline \multicolumn{2}{|l|}{ Sex } \\
\hline Male & $99(53.8)$ \\
\hline Female & $85(46.2)$ \\
\hline Body mass index, $\mathrm{kg} / \mathrm{m}^{2}$, mean (SD) & $26.3(4.1)$ \\
\hline Comorbidity, any & $70(38.0)$ \\
\hline \multicolumn{2}{|l|}{ Performance status (ECOG) } \\
\hline 0 (asymptomatic) & $172(93.5)$ \\
\hline 1 (symptoms, but fully active) & $12(6.5)$ \\
\hline \multicolumn{2}{|l|}{ Smoking status } \\
\hline Never smoker & $75(40.8)$ \\
\hline Former smoker & $15(8.2)$ \\
\hline Current smoker & $72(39.1)$ \\
\hline Unknown & $22(12.0)$ \\
\hline PET-CT preoperatively & $54(29.3)$ \\
\hline \multicolumn{2}{|l|}{ Location, primary tumor } \\
\hline Colon & $37(20.1)$ \\
\hline Sigmoideum & $39(21.2)$ \\
\hline Rectum & $108(58.7)$ \\
\hline \multicolumn{2}{|l|}{ Primary CRC stage } \\
\hline 1 & $9(4.9)$ \\
\hline II & $46(25.0)$ \\
\hline III & $86(46.8)$ \\
\hline IV & $31(16.8)$ \\
\hline Unknown & $12(6.5)$ \\
\hline Preoperative radiotherapy & $4(2.1)$ \\
\hline Preoperative chemotherapy & $41(22.3)$ \\
\hline Other metastases & $91(49.5)$ \\
\hline Liver & $57(31.0)$ \\
\hline Lung & 30 (16.3) \\
\hline Other & $4(2.1)$ \\
\hline \multicolumn{2}{|l|}{ Disease-free interval } \\
\hline$<24$ months & $95(51.6)$ \\
\hline$\geq 24$ months & $88(47.8)$ \\
\hline Unknown & $1(0.5)$ \\
\hline
\end{tabular}

Table 1 (continued)

\begin{tabular}{|c|c|}
\hline Variable & $\mathrm{N}(\%)$ \\
\hline \multicolumn{2}{|l|}{ CEA } \\
\hline$<4 \mu \mathrm{g} / \mathrm{L}$ & $94(51.1)$ \\
\hline$\geq 4 \mu \mathrm{g} / \mathrm{L}$ & $17(9.2)$ \\
\hline Unknown & $73(39.7)$ \\
\hline \multicolumn{2}{|l|}{ Extent of resection } \\
\hline Sublobar resection & $144(78.3)$ \\
\hline Lobectomy & $40(21.7)$ \\
\hline VATS & $85(46.2)$ \\
\hline \multicolumn{2}{|l|}{ Number of metastases } \\
\hline 1 & $141(76.6)$ \\
\hline 2 & $29(15.8)$ \\
\hline 3 or more & $14(7.6)$ \\
\hline \multicolumn{2}{|c|}{ Size of pulmonary metastases (largest lesion) } \\
\hline$<20 \mathrm{~mm}$ & $126(68.5)$ \\
\hline$\geq 20 \mathrm{~mm}$ & $50(27.2)$ \\
\hline Unknown & $8(4.3)$ \\
\hline Hospital stay, days, mean (SD) & $5.5(1.6)$ \\
\hline \multicolumn{2}{|c|}{$\begin{array}{l}\text { CEA, carcinoembryonic antigen; CRC, colorectal cancer; ECOG } \\
\text { Eastern Cooperative Oncology Group; VATS, video-assiste } \\
\text { thoracoscopic surgery; PET-CT, positron emission tomography } \\
\text { computed tomography. }\end{array}$} \\
\hline
\end{tabular}

Table 1. Most patients (93\%) had performance status 0 , i.e., free from symptoms, and $38 \%$ had at least one comorbidity. A positron emission tomography-computed tomography was performed preoperatively in $29 \%$. In $59 \%$, the location of the primary CRC tumor was in the rectum, and $49 \%$ had additional metastases. In $46 \%$, the pulmonary resection was performed by video-assisted thoracoscopic surgery (VATS), and a sublobar resection was done in most patients (78\%).

\section{Number of operations}

The number of operations per year increased during the study period as shown in Figure 1. During the study period, the number of pulmonary metastasectomy procedures increased from 1 in 2004 to 34 in 2015, with a peak of 45 procedures in 2014.

Table 1 (continued) 


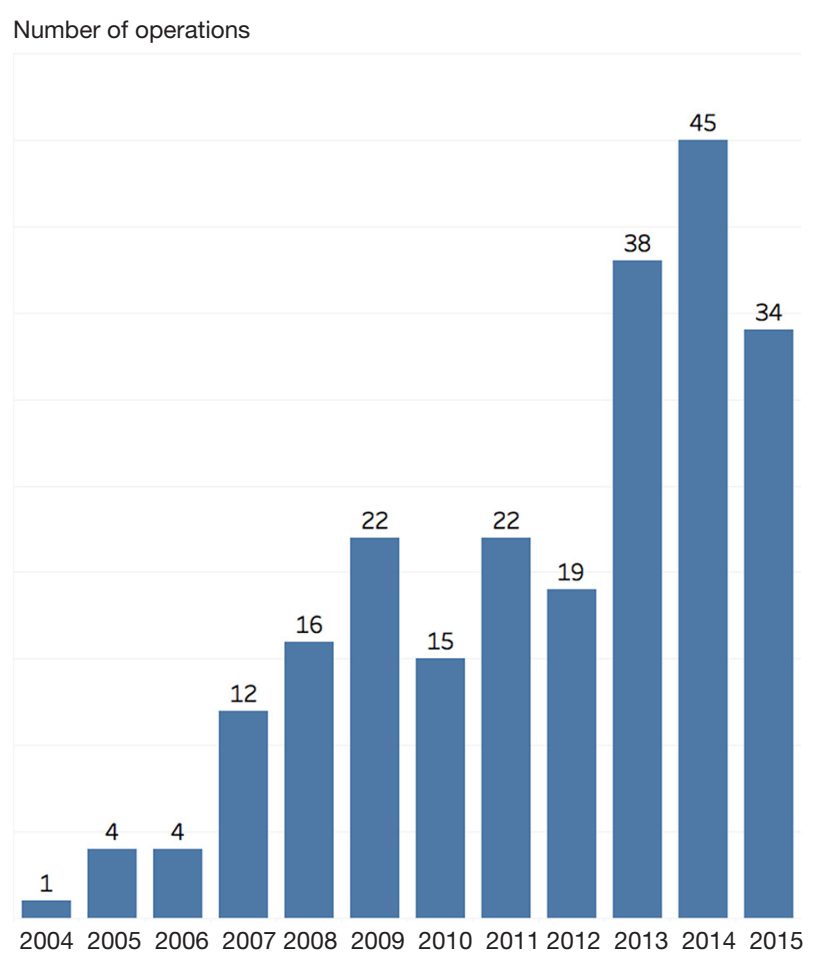

Figure 1 Number of operations (pulmonary resections for colorectal cancer) per year during the study period.

\section{Survival}

During a median follow-up time of 3.2 years, $36 \%$ (66/184) patients died. The Kaplan-Meier estimated survival at 5 years was $60 \%$ (95\% CI: 50-68\%) (Figure 2). Figure 3 shows the observed overall survival and $95 \% \mathrm{CI}$ in patients who underwent pulmonary metastasectomy for CRC compared to the expected survival of an age and gender matched Swedish population. Initially, the survival was similar in the study population and the age and gender matched Swedish population, but after approximately 1 year, the survival in the study population was significantly lower than the matched reference population.

\section{Prognostic factors for survival}

The unadjusted association between selected patient characteristics and mortality is shown in Table 2 and Figure 4. We found a statistically significant association between a CEA level above $4 \mu \mathrm{g} / \mathrm{L}$ (HR, 2.30; 95\% CI: 1.09-4.85, $\mathrm{P}=0.029)$. Other selected characteristics was not significantly

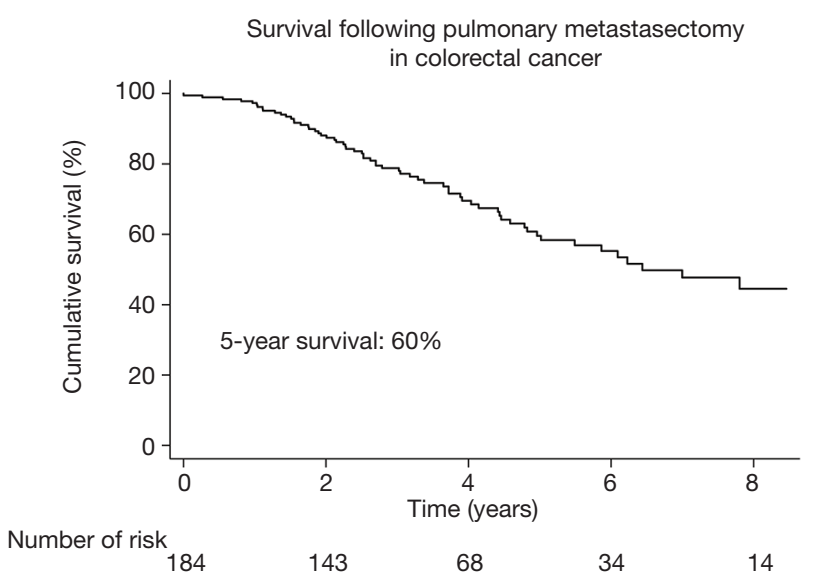

Figure 2 Overall Kaplan-Meier estimated survival in patients who underwent pulmonary metastasectomy for colorectal cancer.

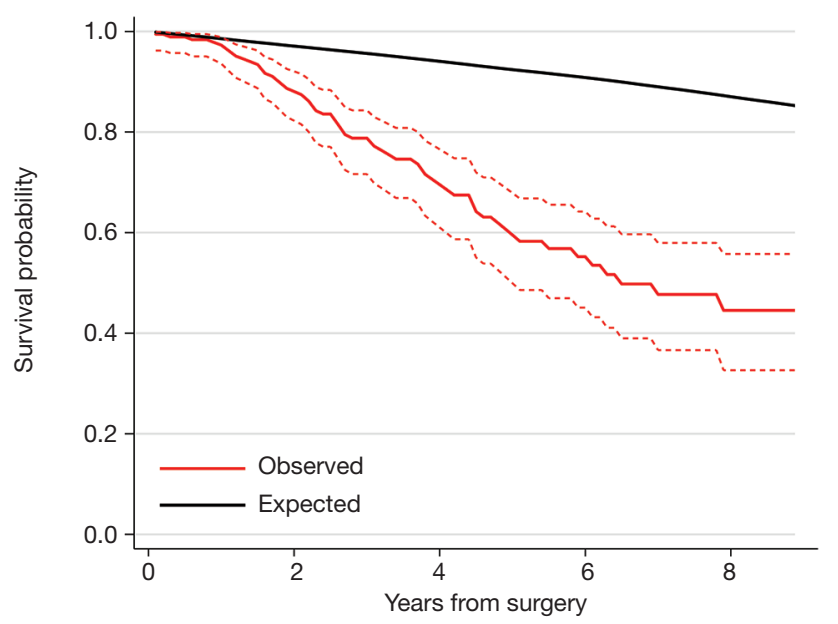

Figure 3 The figure shows the observed overall survival (red line) and $95 \%$ confidence interval (red dashed lines) in patients who underwent pulmonary metastasectomy for colorectal cancer and the expected survival (black line) of an age and gender matched Swedish population.

associated with mortality.

The age and sex adjusted association between selected patient characteristics and mortality is shown in Table 2 . We found a statistically significant association between a CEA level above $4 \mu \mathrm{g} / \mathrm{L}$ and mortality (HR, 2.46; $95 \% \mathrm{CI}$ : 1.15-5.26, $\mathrm{P}=0.020)$. Other selected characteristics was not significantly associated with mortality after adjustment for age and sex. 
Table 2 Prognostic factors for survival in patients who underwent pulmonary metastasectomy after colorectal cancer

\begin{tabular}{|c|c|c|c|c|}
\hline Variable & Crude, HR $(95 \% \mathrm{Cl})$ & $\mathrm{P}$ & Age and sex, adjusted HR (95\% Cl) & $\mathrm{P}$ \\
\hline Female sex & $1.07(0.66-1.73)$ & 0.792 & $1.08(0.67-1.76)^{\star \star}$ & 0.745 \\
\hline Any comorbidity & $1.58(0.97-2.60)$ & 0.069 & $1.56(0.94-2.59)$ & 0.083 \\
\hline Other metastases (liver or lung) & $1.23(0.76-2.00)$ & 0.407 & $1.26(0.77-2.06)$ & 0.361 \\
\hline More than one pulmonary metastasis & $1.39(0.82-2.35)$ & 0.216 & $1.42(0.84-2.41)$ & 0.189 \\
\hline Size of pulmonary metastases $20 \mathrm{~mm}$ or more & $1.00(0.59-1.67)$ & 0.990 & $0.98(0.58-1.65)$ & 0.945 \\
\hline Disease free interval $<24$ months & $1.11(0.68-1.81)$ & 0.668 & $1.14(0.70-1.87)$ & 0.595 \\
\hline CEA above $4 \mu \mathrm{g} / \mathrm{L}$ & $2.30(1.09-4.85)$ & 0.029 & $2.46(1.15-5.26)$ & 0.020 \\
\hline
\end{tabular}

*, only adjusted for sex; **, only adjusted for age. HR, hazard ratio; Cl, confidence interval; CEA, carcinoembryonic antigen.
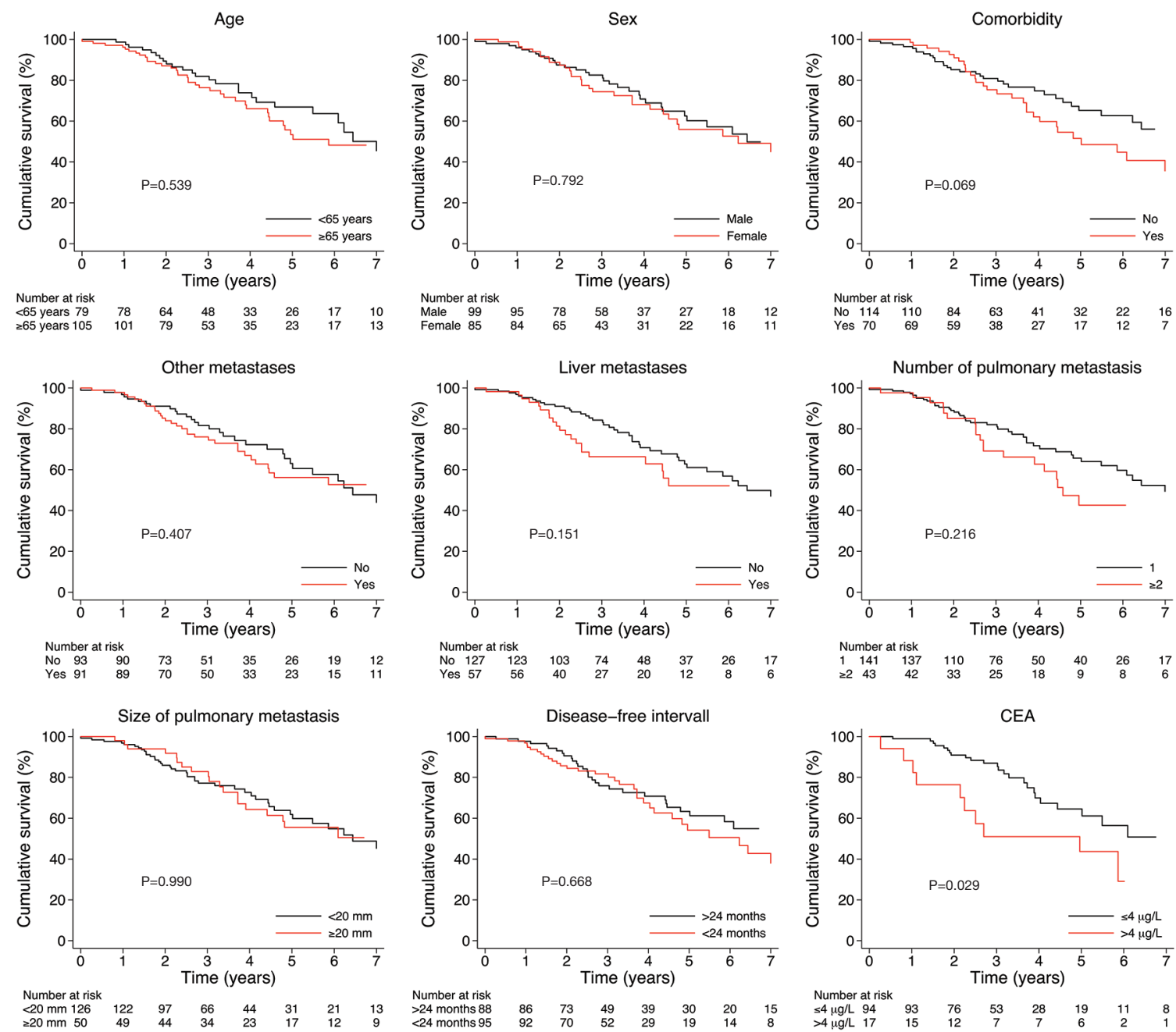

Figure 4 Overall Kaplan-Meier estimated survival in patients who underwent pulmonary metastasectomy for colorectal cancer according to differences in clinically relevant patient characteristics study period. CEA, carcinoembryonic antigen. 


\section{Discussion}

The aims of this study were to characterize the practice of pulmonary metastasectomy at Karolinska University Hospital and to identify prognostic factors that might be of value in clinical decision making, in particular when deciding to offer surgery or not.

During the study period lasting from 2004 to 2015 the number of pulmonary metastasectomy procedures increased considerably. The incidence of CRC in Europe and USA has started to decrease, so this cannot explain the manifold increase of procedures in this relatively short time (15). There has been no formal change of protocol regarding pulmonary metastasectomy at our clinic during the study period. We speculate that this dramatic increase in a relatively short time reflects a general belief among oncologists and thoracic surgeons that aggressive surgery after relapse might be beneficial for the patient in terms of survival and quality of life. It might also be an effect of increased effectiveness and vigilant use of diagnostic methods such as PET-CT scans and measurement of CEAlevels that generates more patients for referral in an earlier stage where surgery might still be an option.

To date no randomized controlled trial has published results to inform and guide clinical practice, and strong evidence supporting surgery is lacking. As discussed by Åberg and colleagues, some of the survival benefit might be attributable to other factors such as selection bias, lead-time bias and staging migration (16). Currently, a randomized controlled trial (the pulmonary metastasectomy in colorectal cancer, PulMiCC trial) is enrolling patients, and the community eagerly awaits its results (7).

In our study, 5 -year survival was $60 \%$. This was in line with previously reported overall survival data after pulmonary metastasectomy. In a systematic review and meta-analysis by Gonzalez et al., overall 5 -year survival ranged from $27 \%$ to $68 \%$ (11). A recent prospective cohort study presented a disease specific 5 -year survival of $46.1 \%$ (95\% CI: $38.5 \%$ to $53.7 \%$ ) (10). The generally small but present differences might be explained in part by different selection criteria for surgery at different centers. If only patients with long DFI, solitary unilateral tumor, negative t-LNs on PET-CT and low CEA are accepted for surgery, the 5 -year survival would most likely be in the upper end. In the study by Embun et al., 69\% of patients had a prethoracotomy CEA of $\leq 5$ and $35 \%$ had a DFI $>24$ months (10). In our study $51 \%$ had a CEA below 4 and $48 \%$ had a DFI $>24$ months. In a retrospective study including
199 patients by Zampino et al., overall 5 -year survival was reported to be $43 \%$. Fifty-two percent had a CEA of $\leq 5$, and $86 \%$ had a DFI of $\geq 12$ months (8). The cut-off for normal $v s$. elevated CEA level at our hospital was $4 \mathrm{ng} / \mathrm{mL}$, although other studies have used a cut-off level of $5 \mathrm{ng} / \mathrm{mL}$ $(17-20)$.

A particular difficult situation can arise with the use of CEA as previously discussed by Fiorentino and colleagues (21). High CEA levels after primary tumor resection might lead to the discovery of lung metastases and referral to thoracic surgery. Then the same test results might be used as a reason to decline surgery due to its negative prognostic value. Prethoracotomy CEA $>4 \mu \mathrm{g} / \mathrm{L}$ was the only prognostic factor that reached statistical significance in our study. In the meta-analysis by Gonzalez et al., 25 studies with a total of 2,925 patients showed a pooled outcome with increased CEA prognostic of an increased risk of death (HR, 1.91; 95\% CI: 1.57-2.32). Thirteen of the included studies reported normal CEA as a prognostic factor for survival (11). In a retrospective study by Suzuki et al. including 94 patients, the only prognostic factor identified in multivariate analysis was elevated prethoracotomy CEA. Sixty-three percent had normal CEA and a 5-year survival of $57 \%$ compared to $30.9 \%$ in the elevated CEA group $(\mathrm{P}=0.038)$ (9). Although high CEA is consistently reported in the literature as a negative prognostic factor it is generally not used as a strict contraindication for surgery. We believe that the role for CEA in pulmonary metastasectomy is primarily in detecting recurrent disease early, thereby increasing lead-time and enable rapid intervention. Until comparative studies such as randomized controlled trials can show effectiveness of surgery, metastasectomy should be performed if radical resection is feasible or if a reduction of symptoms can be achieved.

In our study DFI did not reach statistical significance as a prognostic factor. There are conflicting results in the literature regarding the potential prognostic value of DFI. In the reports by Gonzalez and Embun, a short DFI was prognostic of decreased survival-rate $(10,11)$. However, in several other studies, DFI did not reach statistical significance $(8,9,22)$. It seems reasonable to speculate that a short DFI would reflect a more aggressive disease or a more advanced primary tumor, which in turn would be detrimental to survival. It is possible that our study was underpowered to detect a small but clinically relevant difference in survival between the two groups. 


\section{Limitations}

The size of this study comprises both its main strength and weakness. In general terms, a study with 184 patients cannot be considered a large study with a high likelihood of picking up small intergroup differences. However, in the literature of this subject, many studies contain small patient numbers. In the meta-analysis by Gonzalez et al., 13 of 25 included studies had a study population of less than 100 patients (11). Thus, the findings in our study might be of sufficient power to contribute in future pooled data analyses.

Although not the aim of this study, one of the most important questions in this field that needs an answer is the true effectiveness of pulmonary metastasectomy. This cannot be answered satisfyingly by descriptive retrospective studies, but needs to be approached by randomized controlled trials.

Other limitations of this study are the problem of missing data, bias and confounders inherent in retrospective studies. One example of missing data was prethoracotomy CEA that was unknown in $40 \%$ of the patients.

This might skew the results, however, it is the authors belief that a full data-set on that variable would show a stronger prognostic value, as supported by previous studies discussed above. There was a low frequency of missing data and follow-up regarding survival status was complete. This is due to the high quality Swedish national registers which safeguards against missing information in regards of survival even if the patient moves to a different county or country.

Our institution is the sole practitioner of cardiothoracic surgery in our county so any referrals for recurrent metastatic disease in the lungs would come to us. This results in a low risk for loss to follow-up regarding recurrent surgery. It is possible however that a few patients moved to a different county during the study period and hence was lost to follow-up regarding recurrent disease. It was not possible to determine when and if that was the case from our dataset.

\section{Conclusions}

We found a steady increase in the number of pulmonary metastasectomies after CRC during the study period. We identified prethoracotomy CEA level as a prognostic factor for long-term survival, which was consistent with prior reports. The 5 -year overall survival rate in our study was $60 \%$, which was high in comparison with prior reports. Although our results indicated that current patient selection criteria were reasonable, definitive evidence of efficacy is pending.

\section{Acknowledgements}

Funding: This work was supported by the Mats Kleberg Foundation [2016-00015 to US].

\section{Footnote}

Conflicts of Interest: The authors have no conflicts of interest to declare.

Ethical Statement: The study was approved by the regional Human Research Ethics Committee, Stockholm, Sweden (Dnr: 2014/129-31/1). The need for informed consent was waived by the Ethics Committee.

\section{References}

1. Ferlay J, Soerjomataram I, Dikshit R, et al. Cancer incidence and mortality worldwide: sources, methods and major patterns in GLOBOCAN 2012. Int J Cancer 2015;136:E359-86.

2. Jemal A, Bray F, Center MM, et al. Global cancer statistics. CA Cancer J Clin 2011;61:69-90.

3. Nationellt vårdprogram 2016. Tjock- och ändtarmscancer (Swedish). 2016. Available online: http://www.cancercentrum. se/globalassets/cancerdiagnoser/tjock--och-andtarm-anal/ vardprogram/nvpkolorektalcancer_2016-03-15.pdf. Accessed Jan 20, 2017.

4. Wang CC, Li J. An update on chemotherapy of colorectal liver metastases. World J Gastroenterol 2012;18:25-33.

5. Gonzalez M, Ris HB, Krueger T, et al. Colorectal cancer and thoracic surgeons: close encounters of the third kind. Expert Rev Anticancer Ther 2012;12:495-503.

6. Manfredi S, Lepage C, Hatem C, et al. Epidemiology and management of liver metastases from colorectal cancer. Ann Surg 2006;244:254-9.

7. Treasure T, Fallowfield L, Lees B, et al. Pulmonary metastasectomy in colorectal cancer: the PulMiCC trial. Thorax 2012;67:185-7.

8. Zampino MG, Maisonneuve P, Ravenda PS, et al. Lung metastases from colorectal cancer: analysis of prognostic factors in a single institution study. Ann Thorac Surg 2014;98:1238-45.

9. Suzuki H, Kiyoshima M, Kitahara M, et al. Long-term outcomes after surgical resection of pulmonary metastases 
from colorectal cancer. Ann Thorac Surg 2015;99:435-40.

10. Embun R, Rivas de Andres JJ, Call S, et al. Causal Model of Survival After Pulmonary Metastasectomy of Colorectal Cancer: A Nationwide Prospective Registry. Ann Thorac Surg 2016;101:1883-90.

11. Gonzalez M, Poncet A, Combescure C, et al. Risk factors for survival after lung metastasectomy in colorectal cancer patients: a systematic review and meta-analysis. Ann Surg Oncol 2013;20:572-9.

12. Ludvigsson JF, Otterblad-Olausson P, Pettersson BU, et al. The Swedish personal identity number: possibilities and pitfalls in healthcare and medical research. Eur J Epidemiol 2009;24:659-67.

13. Ludvigsson JF, Almqvist C, Bonamy AK, et al. Registers of the Swedish total population and their use in medical research. Eur J Epidemiol 2016;31:125-36.

14. Engstrom PF, Benson AB, 3rd, Saltz L, et al. Colon cancer. Clinical practice guidelines in oncology. J Natl Compr Canc Netw 2003;1:40-53.

15. Haggar FA, Boushey RP. Colorectal cancer epidemiology: incidence, mortality, survival, and risk factors. Clin Colon Rectal Surg 2009;22:191-7.

16. Åberg T, Treasure T. Analysis of pulmonary metastasis as an indication for operation: an evidence-based approach.
Eur J Cardiothorac Surg 2016;50:792-8.

17. Hachimaru A, Maeda R, Suda T, et al. Repeat pulmonary resection for recurrent lung metastases from colorectal cancer: an analysis of prognostic factors. Interact Cardiovasc Thorac Surg 2016;22:826-30.

18. Hwang MR, Park JW, Kim DY, et al. Early intrapulmonary recurrence after pulmonary metastasectomy related to colorectal cancer. Ann Thorac Surg 2010;90:398-404.

19. Maeda R, Suda T, Hachimaru A, et al. Video-Assisted Thoracoscopic Pulmonary Metastasectomy in Patients with Colorectal Cancer: A Recent 10-Year SingleInstitution Experience. World J Surg 2016;40:1318-23.

20. Sun F, Chen L, Shi M, et al. Prognosis of video-assisted thoracoscopic pulmonary metastasectomy in patients with colorectal cancer lung metastases: an analysis of 154 cases. Int J Colorectal Dis 2017;32:897-905.

21. Fiorentino F, Treasure T. Pulmonary metastasectomy for colorectal cancer: making the case for a randomized controlled trial in the zone of uncertainty. J Thorac Cardiovasc Surg 2013;146:748-52.

22. Cho JH, Kim S, Namgung M, et al. The prognostic importance of the number of metastases in pulmonary metastasectomy of colorectal cancer. World J Surg Oncol 2015;13:222.
Cite this article as: Al-Ameri $\mathrm{M}$, Persson $\mathrm{M}$, Bergman $\mathrm{P}$, Franco-Cereceda A, Sartipy U. Long-term survival after surgery for pulmonary metastases from colorectal cancer: an observational cohort study. J Thorac Dis 2017;9(11):4358-4365. doi: $10.21037 /$ jtd.2017.10.51 